\title{
Introducing EVTM In Turkey
}

\author{
Yunus Emre Özlüer MD ${ }^{1}$ and Mücahit Avcil MD ${ }^{1}$
}

'Department of Emergency Medicine, Adnan Menderes University Hospital, Aydin, Turkey

Trauma is one of the leading causes of death affecting mostly young populations worldwide [1]. Trauma is not only the "sickness of the healthy", but also causes workforce loss in addition to the cost of the healing the trauma victims [2]. Considering the trimodal distribution of the mortality in trauma patients, one can say that trauma is a "syndrome" with a significant rate of mortality and morbidity.

Endovascular trauma management (EVTM) options such as resuscitative endovascular balloon occlusion of the aorta (REBOA), stenting, and embolization are the state-of-the-art tools in the resuscitation of the critically ill trauma patients. However, stenting and embolization require an angiography suite or ideally hybrid suites where REBOA can be placed under the guidance of the ultrasonography making it more accessible even in the austere environments [3].

In Turkey, the health system is mostly carried out through emergency departments (ED) causing an annual ED admittance over 137 million. On the other hand, most of the EDs are not equipped with modern infrastructure (hybrid suites to be specific) to provide up-to-date trauma care. Also, there are not any EDs that are specifically designed for the management of trauma patients.

Trauma surgery is not recognized as a subspecialty/ specialty in Turkey and teamwork is mostly hard to establish by emergency physicians because of the "overconservative approach" of the surgeons. Although the emergency medical services (EMS) are doing an exceptional job, mobile units tailored for the management of the trauma patients on the scene are not available yet. Considering these, one can say that the system has a major part in the high rates of mortality and many unnecessary deaths from trauma.

\section{Corresponding author:}

Yunus Emre Özlüer, Department of Emergency Medicine, Adnan Menderes University Hospital, Aytepe Mevkii, 09100, Efeler, Aydın, Turkey.

Email: yeozluer@adu.edu.tr

(c) 2020 CC BY 4.0 - in cooperation with Depts. of Cardiothoracic/ Vascular Surgery, General Surgery and Anesthesia, Örebro University Hospital and Örebro University, Sweden
The EVTM Symposium in 2018 in Örebro clearly showed us that we needed a paradigm shift in the trauma concept of our country by introducing endovascular resuscitation solutions and ultimately the "endo mind". After experiencing REBOA, stenting, and embolization in the two consecutive EVTM workshops in Örebro, we have analyzed the perspectives of the adaptability of EVTM into our trauma care system. Considering the feasibility and the needs of the system, we thought that REBOA would make a significant difference in the management of trauma patients in Turkey. Thus, we have established the Turkish Endovascular Resuscitation and Trauma Management (TR-EVTM) Platform which stems from our clinic, Adnan Menderes University Hospital Department of Emergency Medicine, designed as a working group to establish a network between EDs and spread the knowledge about REBOA and other endo tools. On the other hand, we have set up a REBOA kit which includes various sizes of introducer sheaths, an 8-Fr Fogarty occlusion catheter, and have started to perform REBOA for the first time in Turkey. To date, we have had 26 REBOA cases. Although REBOA is designed for trauma in the first place, a significant portion of our patients have needed an aortic occlusion on a medical basis. Paying attention to the protection of privacy, in a few of our cases we tried to use social media platforms to draw attention and to prove that REBOA is practically feasible.

By internalizing the motto of the EVTM society, we have started organizing REBOA workshops on live animal models in collaboration with the Emergency Physicians Association of Turkey (EPAT). Members of our platform pledged themselves to promote EVTM at every academic meeting, symposium, and congress they attended. We also had the honor of hosting one of the precious members of the EVTM society, Mr. Yosuke Matsumura, at an emergency medicine congress last year and with a group of committed emergency physicians we have completed the translation of the book "Top Stent" into Turkish by the end of January 2020. Another example of collaboration with colleagues from cardiology at our hospital provided a fantastic opportunity to perform the first pelvic embolization by emergency physicians in a patient with an open book pelvic 
trauma in Turkey. After introducing REBOA in our country, embolizing a bleeding artery was another revolutionary step for EVTM in Turkey.

The industry is a vital part of EVTM. Stent-grafts and embolization materials such as coils and plugs are currently used by interventional radiologists, even in elective cases. However, in Turkey none of the low-profile catheters, which are specifically designed for REBOA, are available on the market. This does not leave any other option other than using large bore, generic catheters. Thanks to the EVTM society, we are increasing our relations with catheter companies and, hopefully, we will be able to have them on the market soon.

TR-EVTM is planning to increase the number and enrich the content of the REBOA workshops where the industry and health professionals come together and share their experiences and knowledge, similar to EVTM workshops. Our main goal is to encourage the use of REBOA in trauma all over the country as a first step. The attendees of our workshops are subscribed to a mailing list (TR-REBOA Registry) designed as a platform for sharing their future REBOA cases. With the collection of data from REBOA cases in Turkey, we will be able to publish our country-wide experience on REBOA. Apart from the workshops, we are planning to hold REBOA courses to a lesser extent than workshops where we will make demonstrations on mannequins rather than live animal models. We think this will provide us with the mobility we need and allow the introduction of REBOA to more people dealing with trauma every day.

\section{Ethics Statement}

(1) All the authors mentioned in the manuscript have agreed to authorship, read and approved the manuscript, and given consent for submission and subsequent publication of the manuscript.

(2) The authors declare that they have read and abided by the JEVTM statement of ethical standards including rules of informed consent and ethical committee approval as stated in the article.

\section{Conflicts of Interest}

The authors declare that they have no conflicts of interest.

\section{Funding}

The authors received no financial support for the research, authorship, and/or publication of this article.

\section{Author Contributions}

YEO and MA both did the literature review and the manuscript writing.

\section{REFERENCES}

[1] Alberdi F, Garcia I, Atutxa L, Zabarte M; Trauma and Neurointensive Care Work Group of The SEMICYUC. Epidemiology of severe trauma. Medicina Intensiva. 2014; 38(9):580-8.

[2] Gabbe BJ, Sleney JS, Gosling CM, et al. Financial and employment impacts of serious injury: a qualitative study. Injury. 2014;45(9):1445-51.

[3] de Schoutheete JC, Fourneau I, Waroquier F, et al. Three cases of resuscitative endovascular balloon occlusion of the aorta (REBOA) in austere pre-hospital environmenttechnical and methodological aspects. World J Emerg Surg. 2018;13:54. 